\title{
Cold-standby redundancy allocation problem with degrading components
}

\author{
Wei Wang ${ }^{\mathrm{a}, \mathrm{b}}$, Junlin Xiong ${ }^{\mathrm{a} *}$ and Min Xie ${ }^{\mathrm{b}}$ \\ ${ }^{a}$ Department of Automation, University of Science and Technology of China, Hefei, China; ${ }^{b}$ Department \\ of Systems Engineering and Engineering Management, City University of Hong Kong, Hong Kong,
} China

(Received 16 June 2014; accepted 9 March 2015)

\begin{abstract}
Components in cold-standby state are usually assumed to be as good as new when they are activated. However, even in a standby environment, the components will suffer from performance degradation. This article presents a study of a redundancy allocation problem (RAP) for cold-standby systems with degrading components. The objective of the RAP is to determine an optimal design configuration of components to maximize system reliability subject to system resource constraints (e.g. cost, weight). As in most cases, it is not possible to obtain a closed-form expression for this problem, and hence, an approximated objective function is presented. A genetic algorithm with dual mutation is developed to solve such a constrained optimization problem. Finally, a numerical example is given to illustrate the proposed solution methodology.
\end{abstract}

Keywords: system reliability; cold-standby; redundancy allocation; degrading performance; genetic algorithm; dual mutation

\section{Introduction}

The development of industrial technologies involves an increasing amount of design of complex and interrelated systems. Reliability is an important performance measure of industrial systems, especially when it is of safety-critical concerns. Allocating redundant components in a system is a popular and direct design methodology of enhancing system reliability (Kuo and Prasad 2000; Kuo and Wan 2007). However, introducing redundant components is a difficult decision due to the limited resources and the economic considerations. Many researchers have studied this redundancy allocation problem (RAP), for example Kumar et al. (2009), Feizollahi and Modarres (2012), Chambari et al. (2013), Laniado and Lillo (2014), Xiao and Peng (2014).

In general, active redundancy has been the main focus in this type of problem. Recently, cold-standby (Coit 2001; van Gemund and Reijns 2012; Levitin, Xing, and Dai 2013a, 2013b; Wang, Xing, and Amari 2012) and warm-standby (Amari, Pham, and Misra 2012; Eryilmaz 2013) have begun to draw more attention. The warm-standby is an intermediate case between active-standby and cold-standby; the standby components operate in a milder environment. In contrast with active-standby, the cold-standby elements are unpowered and thus do not operate until needed to replace a failed online component. The likelihood of failure in the cold-standby state is very low and has usually been assumed to be zero. Therefore, using cold-standby redundancy can usually provide higher system reliability than using active-standby and warm-standby redundancy, especially for large and long-term systems.

*Corresponding author. Email: xiong77@ustc.edu.cn 
In many engineering systems, such as deep-sea exploration, outer-space equipment and other facilities in extreme environment, it is not possible to carry out frequent maintenance and repairs, so the cold-standby redundancy is used to ensure the system works with high reliability in the long term. However, when exposed in certain environments with extreme temperature, humidity, pressure or radiation for a long time, the performance of cold-standby components will decrease more or less. The performance degradation of cold-standby component is not negligible, and it is a complex and irreversible stochastic process, which may ultimately lead to component failure.

Considerable research efforts have been dedicated to studying and formulating degradation or deterioration processes recently. Zhao and Xie (1994) and Zhao, Xie, and Zhang (1995) studied the storage reliability and discussed the failure and deterioration of items in a dormant state. $\mathrm{Lu}$ and Meeker (1993) presented a successful application of degradation modelling to estimate the time-to-failure distribution for a broad class of degradation models. Coit et al. (2005) used degradation modelling to predict reliability and developed a method to correlate field life with observed degradation for electronics modules. Peng, Coit, and Feng (2012) proposed new importance measures as functions of time that can provide timely feedback on the critical components prior to failure based on the measured or observed degradation. Rathod et al. (2012) studied a dynamic reliability-based design optimization model considering probabilistic degradation behaviour, which aimed to capture life cycle issues early at product design and development stage. Furthermore, in the series works of Zhang and Wang (2006, 2007, 2009), deteriorating cold-standby repairable systems were studied using the geometric process. A survey article (Wang 2002) summarized, classified and compared various existing maintenance policies of deteriorating systems.

However, in the aforementioned literature about RAPs and degradation process, none studied the cold-standby RAPs with consideration of the performance degradation of components in the standby state, which is however also a critical factor for the system design and redundancy allocation. Actually, the objective of the RAP is to determine an optimal design configuration to maximize system reliability, but if the system designer only considers the components reliabilities in working state, one can hardly ensure the designed allocation is the optimum one for the whole mission time.

In this article, we consider the reliability of components in the working state as well as the performance degradation of the components in standby state and show how the degrading components affect the result of system design and redundancy allocation. In the cold-standby system, there are several types of choices for each subsystem. Different types of available components have different characteristics and parameters for working state and standby state. Subjected to the cost and resources constraints, the RAP is formulated as a nonlinear constrained integer programming problem. Generally, it is not possible to obtain a closed-form expression for the objective function of this problem, because calculating the multiple integrals and convolutions is a complicated and time-consuming task, especially for the coldstandby systems with components following non-exponential time-to-failure distributions. In Coit (2001), a tractable form has been proposed for cold-standby systems with component time-to-failure following the Erlang distribution. In Rausand and Høyland (2004), the central limit theorem has been used to approximate the distribution functions of renewal process. Following Rausand and Høyland (2004), a fast approximation method has been proposed in Wang, Xing, and Amari (2012) for reliability analysis of cold-standby systems using the central limit theorem. Another method for evaluating the mission reliability of cold-standby system has been presented in Levitin, Xing, and Dai (2013a, 2013b), which is based on a discrete approximation of time-to-failure distributions of system components. In our modelling and analysis, we use an approximation objective function based on the central limit 
theorem. Components with exponential, normal and Weibull-distributed time-to-failure are considered in the modelling.

For solving such a nonlinear constrained integer programming problem, which is NP-hard (Chern 1992), many approaches have been proposed. The most popular approaches in recent are heuristic or meta-heuristic algorithms, such as the genetic algorithm (GA) (Coit and Smith 1996; Tavakkoli-Moghaddam, Safari, and Sassani 2008), hierarchical GA (Kumar et al. 2009), memetic algorithm (Wang, Tang, and Yao 2010), hybrid GA (He et al. 2013), ant colony algorithm (Shelokar, Jayaraman, and Kulkarni 2002), simulated annealing algorithm (Chambari et al. 2013) and particle swarm optimization (Garg and Sharma 2013; Zhang, Wu, and Chen 2014). For more approaches and algorithms, one can refer to the review articles (Kuo and Prasad 2000; Kuo and Wan 2007). In this article, a GA-based approach is developed to solve the considered problem. Penalty function is introduced into the fitness function to handle the constraints, and dual mutation (Wang, Ma, and Wang 2008) is used in the proposed algorithm to avoid premature convergence.

The rest of this article is organized as follows. The next section gives the overview of system model and the formulation of reliability for the cold-standby system with degrading components. Section 3 presents the problem formulation and a detailed solution methodology. In Section 4, a numerical example is used to illustrate the validity of the proposed model and method. Finally, conclusions and future studies are discussed.

\section{Model description}

\subsection{Overview of the model}

We consider a system consisting of $N$ subsystems in series, as shown in Figure 1. The system is functioning only if all the $N$ subsystems are working properly. In subsystem $j$ of the system, there are $m_{j}$ types of components for using and all the $x_{j}$ components in parallel are identical, among them there is one principal functioning component and $x_{j}-1$ cold-standby redundant components.

The following assumptions are made for the system model and used throughout this article,

(1) All the components in the system are statistically independent, and each component has binary states: working and failed.

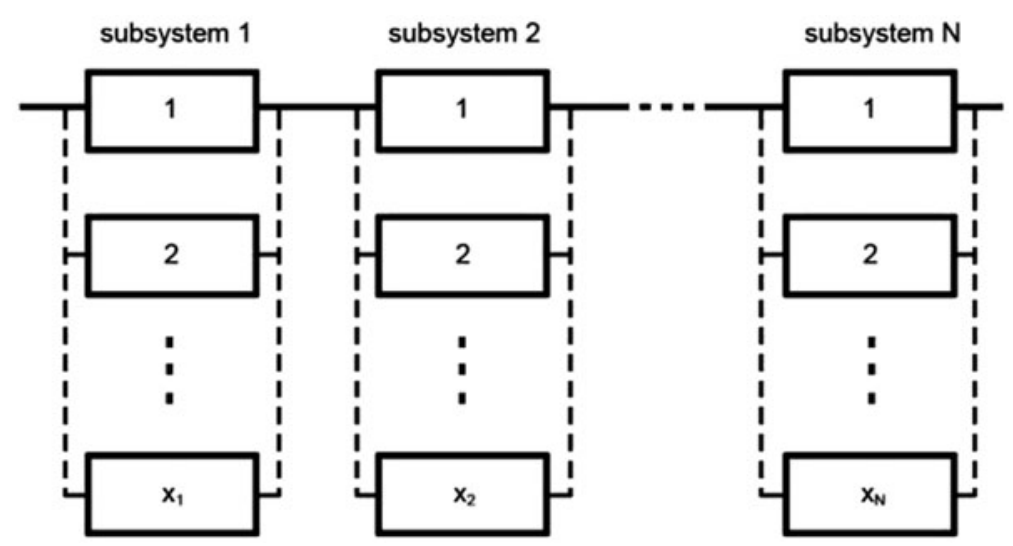

Figure 1. A series system with cold-standby redundant components. 
(2) Components in a subsystem are identical.

(3) The failure detection and component switching mechanisms are fully reliable, and the restoration times are negligible.

(4) Failed components do not damage the system and are not repaired.

In subsystem $j$, all the standby components are used in the specified order, from up to down. The subsystem fails when all the $x_{j}$ components have failed. When component 1 fails at time $t_{1}$, it is replaced by component 2 . When component 2 fails at time $t_{1}+t_{2}$, it is replaced by component 3 , and so on.

The reliability of the cold-standby system without considering performance degradation of cold-standby components is studied in Coit (2001), given as

$$
R(t ; \boldsymbol{z}, \boldsymbol{x})=\prod_{j=1}^{N}\left(r_{j z_{j}}(t)+\sum_{x=1}^{x_{j}-1} \int_{0}^{t} r_{j z_{j}}(t-u) f_{j z_{j}}^{(x)}(u) d u\right), \quad t>0
$$

where $\boldsymbol{z}=\left(z_{1}, z_{2}, \ldots, z_{N}\right) ; \boldsymbol{x}=\left(x_{1}, x_{2}, \ldots, x_{N}\right) ; j=1,2, \ldots, N ; z_{j} \in\left\{1,2, \ldots, m_{j}\right\} ; r_{j z_{j}}(t)$ is the reliability at time $t$ for component of type $z_{j}$ used for subsystem $j ; f_{j z_{j}}^{(x)}(t)$ is the probability density function $(p d f)$ for the $x$ th failure arrival for subsystem $j$.

In Equation (1), if the components have exponential distributed time-to-failure, then a closed-form version of $R(t ; \boldsymbol{z}, \boldsymbol{x})$ can be obtained by considering the failure arrival as a homogeneous Poisson process (Rausand and Høyland 2004). However, for non-exponential distributions, the computation of convolution integrals and $f_{j z_{j}}^{(x)}(t)$ becomes an intractable task.

\subsection{Degrading cold-standby component}

In order to assess the reliability of degrading cold-standby components, the degradation path of component is first determined. The degradation path of a cold-standby component is presented as $\eta(t)=\phi+\Theta t$, where $\phi$ is the initial amount of degradation of the cold-standby component; $\Theta$ represents the degradation rate which is considered following Weibull $(\alpha, \beta)$ distribution ( $\mathrm{Lu}$ and Meeker 1993).

We use $D$ to denote the critical level for the degradation path, above which failure is assumed to have occurred. If the pre-defined failure threshold is $D=\phi+\Theta T$, that is $T=(D-\phi) / \Theta$, then the distribution function of $T$, the time-to-failure in standby state, can be obtained by

$$
F_{T}(t)=\operatorname{Pr}(T \leq t)=\operatorname{Pr}\left(\frac{D-\phi}{\Theta} \leq t\right)=\operatorname{Pr}\left(\Theta \geq \frac{D-\phi}{t}\right)=\exp \left[-\left(\frac{D-\phi}{\alpha t}\right)^{\beta}\right], \quad t>0
$$

In this article, our aim was to determine the optimum redundancy allocation of the cold-standby system, so all the parameters of the degradation model are assumed to be known. Then, Equation (2) can be rewritten as:

$$
F_{T}(t)=\exp \left[-\left(\frac{1}{\lambda^{\prime} t}\right)^{\beta^{\prime}}\right], \quad t>0
$$


where $\beta^{\prime}=\beta, \lambda^{\prime}=\frac{\alpha}{D-\phi}$ and $F_{T}(t)$ is not relevant with the time-to-failure distribution for the working state. Hence, the reliability of a cold-standby component in standby state can be obtained by

$$
\tilde{r}(t)=1-F_{T}(t)=1-\exp \left[-\left(\frac{1}{\lambda^{\prime} t}\right)^{\beta^{\prime}}\right], \quad t>0
$$

\subsection{System reliability with degrading cold-standby redundancy}

In subsystem $j$, the functioning component has a reliability of $r_{j z_{j}}(t)$ and the component in cold-standby state have a reliability of $\tilde{r}_{j z_{j}}(t)$, where $z_{j} \in\left\{1,2, \ldots, m_{j}\right\}$. When the functioning component fails at time $u$, the activated cold-standby component has a reliability of $\tilde{r}_{j z_{j}}(u) r_{j z_{j}}(t-u), t \geq u$. Then the system reliability can be obtained from Equation (1),

$$
R(t ; \boldsymbol{z}, \boldsymbol{x})=\prod_{j=1}^{N}\left(r_{j z_{j}}(t)+\sum_{x=1}^{x_{j}-1} \int_{0}^{t} \tilde{r}_{j z_{j}}(u) r_{j z_{j}}(t-u) \check{f}_{j z_{j}}^{(x)}(u) d u\right), \quad t>0
$$

where $\tilde{r}_{j z_{j}}(t)$ is a non-increasing function; $\check{f}_{j z_{j}}^{(x)}(u)$ is the $p d f$ for the $x$ th failure arrival for subsystem $j$ considering performance degrading of cold-standby components. It is difficult to determine a closed-form version of Equation (5). Therefore, an approximation of system reliability, $\tilde{R}(t ; \boldsymbol{z}, \boldsymbol{x})$, is put forward as follows,

$$
\begin{aligned}
R(t ; \boldsymbol{z}, \boldsymbol{x})= & \prod_{j=1}^{N}\left(r_{j z_{j}}(t)+\sum_{x=1}^{x_{j}-1} \tilde{r}_{j z_{j}}(t) \int_{0}^{t} r_{j z_{j}}(t-u) \check{f}_{j z_{j}}^{(x)}(u) d u\right) \\
& \geq \prod_{j=1}^{N}\left(r_{j z_{j}}(t)+\sum_{x=1}^{x_{j}-1}\left[\tilde{r}_{j z_{j}}(t)\right]^{x+1} \int_{0}^{t} r_{j z_{j}}(t-u) f_{j z_{j}}^{(x)}(u) d u\right)=\tilde{R}(t ; \boldsymbol{z}, \boldsymbol{x}),
\end{aligned}
$$

where $f_{j z_{j}}^{(x)}(t)$ is the $p d f$ for the $x$ th failure arrival for subsystem $j$ without considering performance degrading of cold-standby components. The expression (6) holds because all the replacement of failed components has to operate successfully until the last cold-standby component, that is $\tilde{r}_{j z_{j}}(u) \geq \tilde{r}_{j z_{j}}(t)$ for all $u \leq t$.

The convolution parts in $R(t ; \boldsymbol{z}, \boldsymbol{x})$ are difficult to obtain the exact value. We use the central limit theorem to estimate the convolution parts. The arrival of component failures in subsystem $j$ follows a renewal process $\{N(t), t \geq 0\}$, based on the definition of $N(t)$ and the central limit theorem (Rausand and Høyland 2004; Wang, Xing, and Amari 2012), we can obtain that

$$
\begin{aligned}
\int_{0}^{t} r_{j z_{j}}(t-u) f_{j z_{j}}^{(x)}(u) d u & =\operatorname{Pr}(N(t)=x)=\operatorname{Pr}(N(t) \geq x)-\operatorname{Pr}(N(t) \geq x+1) \\
& \approx \Phi\left(\frac{t-x \mu_{j z_{j}}}{\sigma_{j z_{j}} \sqrt{x}}\right)-\Phi\left(\frac{t-(x+1) \mu_{j z_{j}}}{\sigma_{j z_{j}} \sqrt{x+1}}\right)
\end{aligned}
$$


where $\Phi(\cdot)$ denotes the distribution function of the standard normal distribution $N(0,1) ; \mu_{j z_{i}}$ denotes mean time-to-failure of each component used in subsystem $j ; \sigma_{j z_{j}}$ denotes standard deviation of $T_{i}$ in the count process.

- If the time-to-failure of the component in working state follows exponential distribution with parameter $\lambda_{j z_{j}}$, then in Equation (7); $\mu_{j z_{j}}=\sigma_{j z_{j}}=\frac{1}{\lambda_{j z}}$;

- If the time-to-failure of the component in working state follows normal distribution, $T \sim N\left(v, \tau^{2}\right)$, then in Equation (7), $\mu_{j z_{j}}=v, \sigma_{j z_{j}}=\tau$;

- If the time-to-failure of the component in working state follows Weibull distribution, $T \sim \operatorname{Weibull}\left(\beta_{j z_{j}}, \lambda_{j z_{j}}\right)$, then in Equation (7),

$$
\mu_{j z_{j}}=\frac{1}{\lambda_{j z_{j}}} \Gamma\left(1+\frac{1}{\beta_{j z_{j}}}\right), \sigma_{j z_{j}}=\frac{1}{\lambda_{j z_{j}}} \sqrt{\Gamma\left(1+\frac{2}{\beta_{j z_{j}}}\right)-\left[\Gamma\left(1+\frac{1}{\beta_{j z_{j}}}\right)\right]^{2}},
$$

where $\Gamma(\cdot)$ is Gamma function, $\Gamma(z)=\int_{0}^{\infty} x^{z-1} e^{-x} d x$.

\section{Problem formulation and solution algorithm}

\subsection{Problem formulation}

The purpose of the considered RAP is to determine the optimal combination of component choices, $\boldsymbol{z}$, and the number of redundant components, $\boldsymbol{x}$, and to maximize the system reliability while meeting the resource constraints. So, the RAP for the cold-standby system can be formulated with an objective function of approximated system reliability,

$$
\begin{array}{ll}
\max & \tilde{R}(t ; \boldsymbol{z}, \boldsymbol{x}) \\
\text { s.t. } & \sum_{j=1}^{N} g_{i j}\left(x_{j}\right) \leq b_{i}, \quad \forall i \in I \\
& z_{j} \in\left\{1,2, \ldots, m_{j}\right\}, \quad j=1,2, \ldots, N, \\
& 1 \leq x_{j} \leq \text { maxsize }: \text { integer. }
\end{array}
$$

where $g_{i j}(\cdot)$ is a convex function of $x_{j}$ and the coefficients in $g_{i j}(\cdot)$ are determined by the type of components $z_{j}, I$ is the set of all resource constraints, and $b_{i}$ is the maximum value of the $i$ th resource constraints.

\subsection{GA-based approach}

In order to solve the nonlinear constrained integer programming problem (8), a GA-based approach is developed. In the algorithm, penalty function is used to handle the constraint conditions. The penalty fitness function is presented by,

$$
\text { fitness }(\boldsymbol{z}, \boldsymbol{x})=\tilde{R}(t ; \boldsymbol{z}, \boldsymbol{x})-\sum_{\boldsymbol{i} \in \boldsymbol{I}} \delta_{\boldsymbol{i}} \cdot\left|\sum_{\boldsymbol{j}=\boldsymbol{1}}^{\boldsymbol{N}} \boldsymbol{g}_{\boldsymbol{i j}}\left(\boldsymbol{x}_{\boldsymbol{j}}\right)-\boldsymbol{b}_{\boldsymbol{i}}\right|^{\boldsymbol{k}},
$$

where

$$
\delta_{i}=\left\{\begin{array}{cc}
1, & \text { if constraint } i \text { is violated } \\
0, & \text { otherwise }
\end{array}\right.
$$


and the exponent $\kappa$ is a preset amplification parameter, which is set to 2 throughout this article.

Following Tavakkoli-Moghaddam, Safari, and Sassani (2008), the solution of the problem is encoded as a $2 \times N$ matrix, which is called the chromosome. The two rows represent the type and the quantity of selected components, respectively. A random crossover mask is used in the crossover operator as shown in Figure 2. The crossover mask is a binary matrix of the same size with the chromosome. All $1 \mathrm{~s}$ in the crossover mask mean exchanging the genes of parents in the same positions, and all $0 \mathrm{~s}$ mean no action. The mutation operator performs random perturbations to the selected chromosomes. Each value within the chromosome matrix is randomly altered at a mutation probability of $p_{m}$. As depicted in Figure 3, a $2 \times N$ matrix is generated randomly with all its elements in $(0,1)$, the gene of the selected chromosome in the position, where the value is lower than $p_{m}$, will be altered.

Premature convergence is a noticeable phenomenon in standard GA. In order to avoid the phenomenon and speed up the search, dual mutation (Wang, Ma, and Wang 2008) is introduced into the algorithm, which involves a local mutation and a global mutation. When the population approaches convergence, the Hamming distance between individuals becomes smaller. The Hamming distance between two individuals (of the same length) is the number of places where they differ (Ventura and Yoon 2013), and it is normalized so that the biggest value in the search domain of the problem is equal to 1 . When a pair of parents is randomly selected from the population, their Hamming distance will be calculated. The crossover operator executes only if their Hamming distance is greater than a certain threshold (usually 0.1 0.2 ). Otherwise, the parents will undergo random perturbations with local mutation probability, which is lower than the global mutation probability.

\subsection{Solution algorithm}

The main algorithmic procedures are shown in Algorithm 1.

Algorithm 1. General Framework of GA used to solve the proposed RAP

(1) Generate an initial population $V_{\text {pop }}$ with a population size of pop_size. Set a maximum generation of max_gen, crossover probability $p_{c}$, dual mutation probabilities $p_{m 1}$ and $p_{m 2}$;

(2) Calculate the fitness of all individuals in $V_{p o p}$, preserve the best individual;

(3) Perform roulette selection on $V_{p o p}$, set the selected the group as $V_{\text {sel }}$;

(4) Select a group of individuals from the $V_{\text {sel }}$ with probability of $p_{c}$, the group size is $p \_s i z e$. For any two individuals in the group, calculate their Hamming distance, if greater than a pre-set threshold (0.2), perform crossover operation;

$$
\begin{aligned}
& {\left[\begin{array}{llllll}
3 & 1 & 2 & 3 & 1 & 3 \\
1 & 4 & 2 & 2 & 2 & 1
\end{array}\right] \quad\left[\begin{array}{llllll}
3 & 2 & 2 & 3 & 3 & 1 \\
4 & 4 & 2 & 3 & 2 & 1
\end{array}\right]} \\
& \underset{\operatorname{mask}:\left[\begin{array}{llllll}
0 & 1 & 0 & 0 & 1 & 1 \\
1 & 0 & 0 & 1 & 0 & 1
\end{array}\right]}{\stackrel{\longrightarrow}{\longrightarrow}} \\
& {\left[\begin{array}{llllll}
3 & 2 & 1 & 2 & 3 & 1 \\
4 & 3 & 4 & 3 & 2 & 1
\end{array}\right] \quad\left[\begin{array}{llllll}
3 & 1 & 1 & 2 & 1 & 3 \\
1 & 3 & 4 & 2 & 2 & 1
\end{array}\right]}
\end{aligned}
$$

Figure 2. Crossover operator. 


$$
\left[\begin{array}{llllll}
3 & 1 & 2 & 3 & 1 & 3 \\
1 & 4 & 2 & 2 & 2 & 1
\end{array}\right] \stackrel{\left[\begin{array}{cccccc}
\mathbf{0 . 0 7} & 0.53 & 0.93 & 0.56 & \mathbf{0 . 0 1} & 0.35 \\
0.56 & \mathbf{0 . 0 5} & 0.77 & 0.12 & 0.46 & 0.33
\end{array}\right]}{\underset{7}{\longrightarrow}=0.1}\left[\begin{array}{llllll}
\mathbf{2} & 1 & 2 & 3 & \mathbf{3} & 3 \\
1 & \mathbf{1} & 2 & 2 & 2 & 1
\end{array}\right]
$$

Figure 3. Mutation operator.

(5) Put individuals generated from crossover operation into a temporary population $V_{\text {temp }}$. If the size of $V_{\text {temp }}$ is greater than $p_{-}$size, goto Step 6; Otherwise, perform local mutation operation on $V_{\text {temp }}$ with probability of $p_{m 2}$, then goto Step 4;

(6) Select $p \_$size best individuals from $V_{\text {temp }}$ according to their fitness values, put them back into $V_{\text {sel }}$, then clean $V_{\text {temp }}$;

(7) Perform global mutation operation on $V_{s e l}$ with global mutation probability of $p_{m 1}$;

(8) Use the best individual in Step 2 to replace the worst individual in the $V_{\text {sel }}$;

(9) Let $V_{\text {sel }}$ be the initial population of next generation;

(10) If the number of generations achieves max_gen, the algorithm is terminated, output the final results; Otherwise, goto Step 2.

\section{Numerical example}

In this section, a numerical example is presented to illustrate the considered problem and the proposed solution methodology. The example is based on a similar example presented by Zhang, Wu, and Chen (2014). The mathematical model of the RAP at time $t$ is represented as follows:

$$
\begin{array}{ll}
\max & \tilde{R}(t ; \boldsymbol{z}, \boldsymbol{x}) \\
\text { s.t. } & \sum_{j=1}^{3} c_{j z_{j}} \cdot x_{j} \leq C, \\
& \sum_{j=1}^{3} w_{j z_{j}} \cdot x_{j} \leq W, \\
& z_{j} \in\left\{1,2, \ldots, m_{j}\right\}, \quad j=1,2, \ldots, N, \\
& 1 \leq x_{j} \leq 6: \text { integer. }
\end{array}
$$

The system consists of 3 subsystems, each including 4 or 5 choices for component types, and the maximum number of components allowed in a subsystem is 6. Components in this example are assumed as having exponential distributed time-to-failure in working state and suffering performance degradation before being used. The cost, weight and pre-set distribution parameters of components are all listed in Table 1.

The maximum system cost is $C=38$ and the maximum system weight is $W=32$. The computation of this problem is performed with following initial parameters: population size pop_size $=100$, maximum generation max_gen $=50$, crossover probability $p_{c}=0.95$, global mutation probability $p_{m 1}=0.45$ and local mutation probability $p_{m 2}=0.05$.

Due to the stochastic nature of GA, the proposed Algorithm 1 is performed 10 times and the best one is selected as the final solution. Figure 4 demonstrates the convergence process of the proposed GA to the best compromise solution at time $t=100$. As shown in Figure 4, the optimal solution is achieved at approximately 5 generations. The configuration of the best 


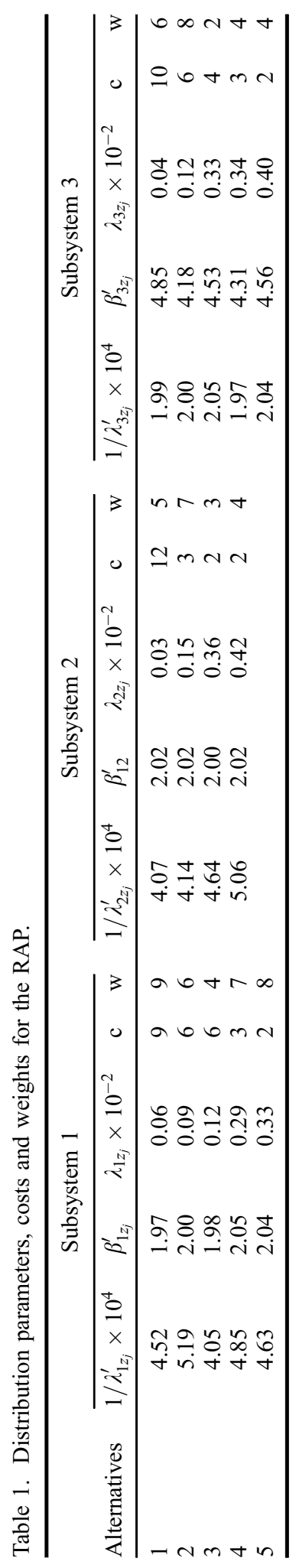




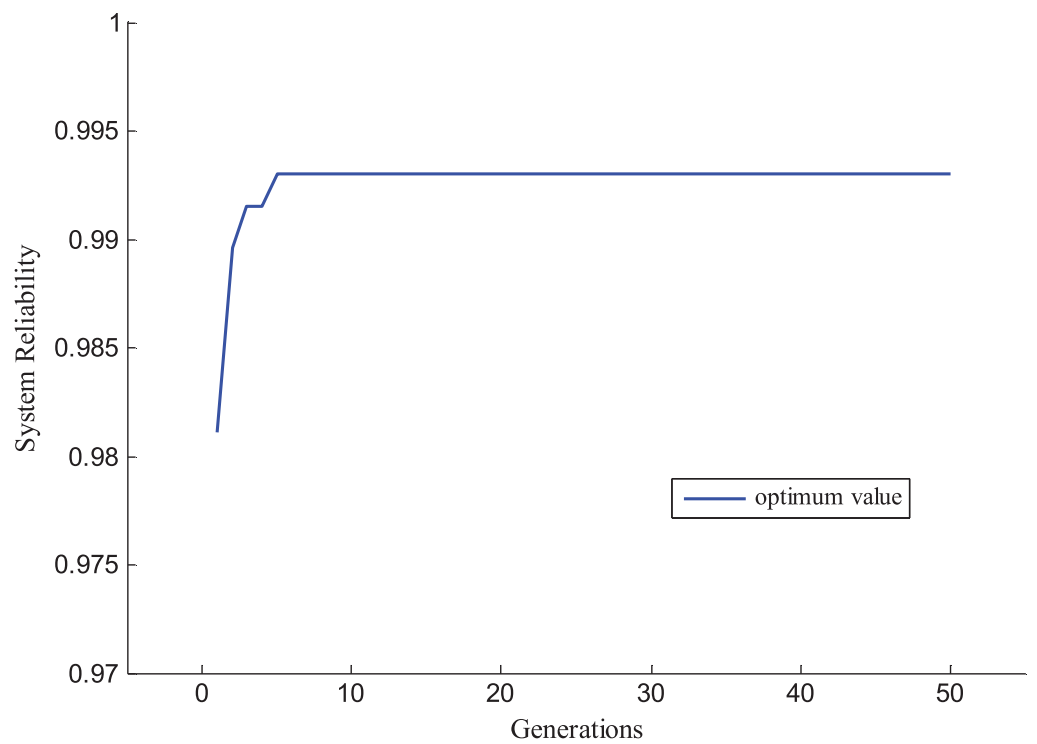

Figure 4. Convergence process for proposed RAP, when $t=100$.

Table 2. Example results.

\begin{tabular}{lccccc}
\hline \multirow{2}{*}{$\begin{array}{l}\text { Subsystem } \\
j\end{array}$} & \multicolumn{2}{c}{ Proposed model } & & \multicolumn{2}{c}{ Comparative trial $^{*}$} \\
\cline { 2 - 6 } & $z_{j}$ & & $x_{j}$ & & $z_{j}$ \\
\hline 1 & 2 & & 2 & 3 & 3 \\
2 & 1 & & 2 & 3 & 5 \\
3 & 5 & & 1 & & $0.9900\left(0.9997^{*}\right)$ \\
Reliability & & 0.9930 & & & 2 \\
Cost & 32 & & & 36 \\
Weight & & 32 & & & 31 \\
\hline
\end{tabular}

*Without considering performance degradation of standby components.

allocated solution of problem (9) is shown in Table 2. The corresponding approximate system reliability is 0.9930 . Total system cost of the optimal solution is 32 , and the total system weight is 32 .

In addition, for comparative purpose, Table 2 also presents the solution of a comparative trail without considering performance degradation of cold-standby components before being activated. In the comparative trail, the best solution corresponds to the system reliability of 0.9997 , the system cost of 36 and the weight of 31 . If system designers neglect the performance degradation of cold-standby components, the solution of comparative trail in Table 2 may be selected. Actually, the best solution of comparative trail only has a reliability of 0.9900 in the model of considering degrading cold-standby components. Evidently, the performance degradation of cold-standby components has dramatic effects on the result of the RAP and the system reliability. Thus, the performance degradation of components in cold-standby state should be considered as a significant factor when allocating redundancy for cold-standby systems. 


\section{Conclusions}

In this article, we studied the redundancy allocation optimization for cold-standby systems. As in the real world, performance of components in standby state may decrease over time, so we should consider the performance degradation of cold-standby redundant components as a factor for redundancy allocation optimization. However, most of the existing models have assumed that the cold-standby components are as good as new before they are activated. In this article, we formulate the reliability of the cold-standby system with consideration of degrading cold-standby components and develop the constraint RAP model. Then, using the central limit theorem, an approximate objective function is put forward to solve the RAP more efficiently. For problem solution, a GA-based approach with dual mutation is developed. Finally, the results of numerical example have validated that considering performance degradation of cold-standby components gives us the real optimal redundancy allocation designs.

Further work includes relaxing some of the assumptions made in this article. Improvements could also be obtained by considering preventive maintenance and inspection for the components.

\section{Disclosure statement}

No potential conflict of interest was reported by the authors.

\section{Funding}

The work described in this article was partially supported by the National Natural Science Foundation of China [grant number 61374026]; the Programme for New Century Excellent Talents in University [grant number 11-0880]; the Fundamental Research Funds for the Central Universities [grant number WK2100100013]; and a grant from City University of Hong Kong [grant number 9380058].

\section{Notes on contributors}

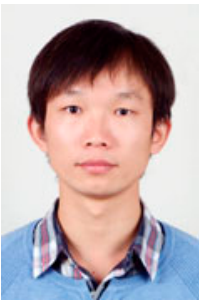

Wei Wang received his BEng degree from the University of Science and Technology of China in 2011. He is currently pursuing his $\mathrm{PhD}$ degree in a joint $\mathrm{PhD}$ programme offered by the University of Science and Technology of China and City University of Hong Kong. His research interests include system reliability modelling and optimization.

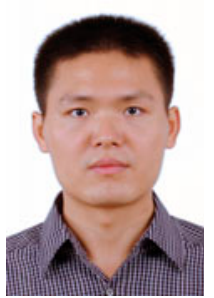

Junlin Xiong received his BEng and MSci degrees from Northeastern University, China, and his $\mathrm{PhD}$ degree from the University of Hong Kong, Hong Kong, in 2000, 2003, and 2007, respectively. From November 2007 to February 2010, he was a research associate at the University of New South Wales at the Australian Defence Force Academy, Australia. In March 2010, he joined the University of Science and Technology of China where he is currently a professor in the Department of Automation. His current research interests are in the fields of control theory and systems reliability analysis. 


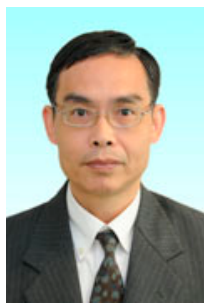

Min Xie received his $\mathrm{PhD}$ in Quality Technology in 1987 from Linkoping University in Sweden. Prior to that, he did his undergraduate study and received his MSc in 1984 from Royal Institute of Technology, Sweden. He is currently with City University of Hong Kong as Chair Professor of Industrial Engineering. He was awarded the prestigious LKY research fellowship in 1991, the same year he joined the National University of Singapore. He has authored or co-authored numerous refereed journal articles and eight books on quality and reliability engineering, including Software Reliability Modelling with World Scientific Publisher, Weibull Models with John Wiley, Computing Systems Reliability with Kluwer Academic and Advanced QFD Applications with ASQ Quality Press. He is an Editor, Associate Editor or on the editorial board of many international journals. Prof. Xie was elected a fellow of IEEE in 2005 for his contribution to software and system reliability.

\section{References}

Amari, S. V., H. Pham, and R. B. Misra. 2012. "Reliability Characteristics of k-out-of-n Warm Standby Systems." IEEE Transactions on Reliability 61 (4): 1007-1018.

Chambari, A., A. A. Najafi, S. H. A. Rahmati, and A. Karimi. 2013. "An Efficient Simulated Annealing Algorithm for the Redundancy Allocation Problem with a Choice of Redundancy Strategies." Reliability Engineering \& System Safety 119: 158-164.

Chern, M. 1992. "On the Computational Complexity of Reliability Redundancy Allocation in a Series System." Operations Research Letters 11 (5): 309-315.

Coit, D. W. 2001. "Cold-standby Redundancy Optimization for Nonrepairable Systems." IIE Transactions 33 (6): 471-478.

Coit, D. W., J. L. Evans, N. T. Vogt, and J. R. Thompson. 2005. "A Method for Correlating Field Life Degradation with Reliability Prediction for Electronic Modules." Quality and Reliability Engineering International 21 (7): 715-726.

Coit, D. W., and A. E. Smith. 1996. "Reliability Optimization of Series-parallel Systems Using a Genetic Algorithm." IEEE Transactions on Reliability 45 (2): 254-260, 266.

Eryilmaz, S. 2013. "Reliability of a k-out-of-n System Equipped with a Single Warm Standby Component." IEEE Transactions on Reliability 62 (2): 499-503.

Feizollahi, M. J., and M. Modarres. 2012. "The Robust Deviation Redundancy Allocation Problem with Interval Component Reliabilities." IEEE Transactions on Reliability 61 (4): 957-965.

Garg, H., and S. Sharma. 2013. "Multi-objective Reliability-redundancy Allocation Problem Using Particle Swarm Optimization." Computers \& Industrial Engineering 64 (1): 247-255.

van Gemund, A. J., and G. L. Reijns. 2012. "Reliability Analysis of k-out-of-n systems with Single Cold Standby Using Pearson Distributions." IEEE Transactions on Reliability 61 (2): 526-532.

He, P., K. Wu, J. Xu, J. Wen, and Z. Jiang. 2013. "Multilevel Redundancy Allocation Using Two Dimensional Arrays Encoding and Hybrid Genetic Algorithm." Computers \& Industrial Engineering 64 (1): 69-83.

Kumar, R., K. Izui, M. Yoshimura, and S. Nishiwaki. 2009. "Optimal Multilevel Redundancy Allocation in Series and Series-parallel Systems." Computers \& Industrial Engineering 57 (1): 169-180.

Kuo, W., and V. R. Prasad. 2000. "An Annotated Overview of System-reliability Optimization." IEEE Transactions on Reliability 49 (2): 176-187.

Kuo, W., and R. Wan. 2007. "Recent Advances in Optimal Reliability Allocation." In Computational Intelligence in Reliability Engineering, edited by G. Levitin, 1-36. Berlin: Springer.

Laniado, H., and R. E. Lillo. 2014. "Allocation Policies of Redundancies in Two-parallel-series and Two-series-parallel Systems.” IEEE Transactions on Reliability 63 (1): 223-229.

Levitin, G., L. Xing, and Y. Dai. 2013a. "Cold-standby Sequencing Optimization Considering Mission Cost." Reliability Engineering \& System Safety 118: 28-34.

Levitin, G., L. Xing, and Y. Dai. 2013b. "Sequencing Optimization in k-out-of-n Cold-standby Systems Considering Mission Cost." International Journal of General Systems 42 (8): 870-882.

Lu, C. J., and W. O. Meeker. 1993. "Using Degradation Measures to Estimate a Time-to-failure Distribution." Technometrics 35 (2): 161-174.

Peng, H., D. W. Coit, and Q. Feng. 2012. "Component Reliability Criticality or Importance Measures for Systems with Degrading Components." IEEE Transactions on Reliability 61 (1): 4-12. 
Rathod, V., O. P. Yadav, A. Rathore, and R. Jain. 2012. "Reliability-based Design Optimization Considering Probabilistic Degradation Behavior." Quality and Reliability Engineering International 28 (8): 911-923.

Rausand, M., and A. Høyland. 2004. System Reliability Theory: Models, Statistical Methods, and Applications. Hoboken, NJ: John Wiley.

Shelokar, P. S., V. Jayaraman, and B. Kulkarni. 2002. "Ant Algorithm for Single and Multiobjective Reliability Optimization Problems." Quality and Reliability Engineering International 18 (6): 497514.

Tavakkoli-Moghaddam, R., J. Safari, and F. Sassani. 2008. "Reliability Optimization of Series-parallel Systems with a Choice of Redundancy Strategies Using a Genetic Algorithm." Reliability Engineering \& System Safety 93 (4): 550-556.

Ventura, J. A., and S.-H. Yoon. 2013. "A New Genetic Algorithm for Lot-streaming Flow Shop Scheduling with Limited Capacity Buffers." Journal of Intelligent Manufacturing 24 (6): 11851196.

Wang, H. 2002. "A Survey of Maintenance Policies of Deteriorating Systems." European Journal of Operational Research 139 (3): 469-489.

Wang, J., Y. Ma, and F. Wang. 2008. "Study of Improved Genetic Algorithm Based on Dual Mutation and Its Simulation." Computer Engineering and Applications 44 (3): 57-59, 90.

Wang, Z., K. Tang, and X. Yao. 2010. "A Memetic Algorithm for Multi-level Redundancy Allocation." IEEE Transactions on Reliability 59 (4): 754-765.

Wang, C., L. Xing, and S. V. Amari. 2012. "A Fast Approximation Method for Reliability Analysis of Cold-standby Systems.” Reliability Engineering \& System Safety 106: 119-126.

Xiao, H., and R. Peng. 2014. "Optimal Allocation and Maintenance of Multi-state Elements in Seriesparallel Systems with Common Bus Performance Sharing." Computers \& Industrial Engineering 72: $143-151$.

Zhang, Y. L., and G. J. Wang. 2006. "A Bivariate Optimal Repair-replacement Model Using Geometric Processes for a Cold Standby Repairable System.” Engineering Optimization 38 (5): 609-619.

Zhang, Y. L., and G. J. Wang. 2007. "A Deteriorating Cold Standby Repairable System with Priority in Use." European Journal of Operational Research 183 (1): 278-295.

Zhang, Y. L., and G. J. Wang. 2009. "A Geometric Process Repair Model for a Repairable Cold Standby System with Priority in Use and Repair." Reliability Engineering \& System Safety 94 (11): $1782-1787$.

Zhang, E., Y. Wu, and Q. Chen. 2014. "A Practical Approach for Solving Multi-objective Reliability Redundancy Allocation Problems Using Extended Bare-bones Particle Swarm Optimization." Reliability Engineering \& System Safety 127: 65-76.

Zhao, M., and M. Xie. 1994. "A Model of Storage Reliability with Possible Initial Failures." Reliability Engineering \& System Safety 43 (3): 269-273.

Zhao, M., M. Xie, and Y. Zhang. 1995. "A Study of a Storage Reliability Estimation Problem." Quality and Reliability Engineering International 11 (2): 123-127. 